\title{
Systemic administration of oxytocin reduces basal and lipopolysaccharide-induced ghrelin levels in healthy men
}

\author{
Greisa Vila, Michaela Riedl, Michael Resl, Aart Jan van der Lely ${ }^{1}$, Leo J Hofland ${ }^{1}$, Martin Clodi \\ and Anton Luger
}

Division of Endocrinology and Metabolism, Department of Internal Medicine III, Medical University of Vienna, Waehringer Guertel 18-20, A-1090 Vienna, Austria

${ }^{1}$ Division of Endocrinology, Department of Internal Medicine, Erasmus University Medical Center, 3000 CA Rotterdam, The Netherlands

(Correspondence should be addressed to G Vila; Email: greisa.vila@meduniwien.ac.at)

\begin{abstract}
Oxytocin (OXT) and ghrelin have several common properties such as the involvement in the first phase response to stressors, in appetite regulation, and in the modulation of neural functions. Despite a recent study showing that intraventricular administration of ghrelin activates OXT neurons, little is known on the cross-talk between these two peptides. Here, we investigated the role of the i.v. administration of OXT on circulating ghrelin concentrations under fasting conditions and during the lipopolysaccharide (LPS)-induced endotoxemia. A randomized placebo-controlled cross-over study was performed in ten healthy men. In four study sessions, the participants received once placebo, once OXT (1 pmol/ $\mathrm{kg}$ per min over $90 \mathrm{~min}$ ), once LPS $(2 \mathrm{ng} / \mathrm{kg})$, and once both OXT and LPS. Plasma
\end{abstract}

ghrelin, glucose, and free fatty acid (FFA) levels were measured at regular intervals during the first $6 \mathrm{~h}$ following the LPS bolus. Systemic administration of OXT decreased within $1 \mathrm{~h}$ plasma ghrelin levels $(611 \pm 54$ vs 697 $\pm 52 \mathrm{pg} / \mathrm{ml}$ in placebo days, $P=0.013)$ and increased plasma glucose and FFA concentrations $(P=0.002$ and $P=0.005$ respectively). OXT also reduced the LPS-induced surge in ghrelin at time point $2 \mathrm{~h}(P=0 \cdot 021)$. In summary, i.v. administration of OXT decreases circulating levels of ghrelin during fasting, as well as following LPS-induced endotoxemia in healthy men. The cross-talk between OXT and ghrelin might be important in the regulation of energy homeostasis and stress responses.

Journal of Endocrinology (2009) 203, 175-179

\section{Introduction}

The interaction between the nervous and endocrine systems is crucial in coordinating physiological processes and the response to stressors (Charmandari et al. 2005, de Kloet et al. 2005). During the last decade, a new neuroendocrine axis has been established: the gut-brain axis (Murphy et al. 2006). Gut-brain signaling consists of bidirectional cross-talks between the hypothalamic nuclei and neuroendocrine cells of the gut, partially mediated by afferent and efferent nerval components (Hosoda \& Kangawa 2008, Maier et al. 2008). This axis controls energy homeostasis and was recently found to be involved in the modulation of stress responses (Murphy et al. 2006, Lutter et al. 2008).

The most important member of the gut-brain axis is ghrelin, a hormone that promotes appetite and growth (Korbonits et al. 2004, van der Lely et al. 2004). Ghrelin immunoreactivity is mainly found in the neuroendocrine cells of the gastric mucosa, and to a lesser extent in the hypothalamus (Kojima et al. 1999). In the hypothalamus, ghrelin is expressed in neurons that lie upstream to neuropeptide Y (NPY) and proopiomelanocortin neurons, suggesting local effects in the regulation of food intake and energy expenditure (van der Lely et al. 2004).

A recent study has showed that intraventricular administration of ghrelin increases the c-fos activity of oxytocin (OXT)-secreting neurons (Olszewski et al. 2007). OXT is involved in social bonding, has important reproductive functions, and is a classical stress hormone (Gimpl \& Fahrenholz 2001, Landgraf \& Neumann 2004). Plasma OXT displays a distinct circadian rhythm and changes during stress response, parturition and lactation, as well as upon pharmacological use of OXT (Windle et al. 1992, Landgraf \& Neumann 2004). OXT infusion in men decreases the NPY response to ghrelin, but an eventual modification of ghrelin levels has not been tested (Coiro et al. 2008).

OXT and ghrelin are hormones with partial but not classical neurotransmitter properties (van der Lely et al. 2004, Ludwig \& Leng 2006). Both increase in response to stressors (Van de Kar \& Blair 1999, Vila et al. 2007, Lutter et al. 2008), thereby exerting anti-inflammatory and anxiolytic effects (Dixit et al. 2004, Landgraf \& Neumann 2004, Clodi et al. 2008, Lutter et al. 2008). These peptides have opposing effects on appetite regulation, OXT being an anorexigenic 
hormone, while ghrelin an orexigenic one (Landgraf \& Neumann 2004, van der Lely et al. 2004). The mechanisms underlying the appetite-promoting effects of ghrelin have been investigated in detail (Cummings et al. 2001, López et al. 2008). The mechanisms underlying the anorexigenic effect of OXT remain unknown, and could also involve components of the gut-brain axis.

We aimed to test whether alterations in circulating OXT modify ghrelin levels under physiological conditions and in response to stressors. This was investigated in a randomized placebo-controlled cross-over study where OXT was intravenously administered under two conditions: during fasting and in response to endotoxemic stress in healthy men.

\section{Subjects and Methods}

\section{Study participants}

The study was approved by the Ethics Committee of the Medical University of Vienna and performed in compliance with the Declaration of Helsinki and Good Clinical Practice guidelines (Trial registration: ClinicalTrials.gov NCT00360048). Ten healthy men aged 20-40 years and with a mean body mass index of $23 \mathrm{~kg} / \mathrm{m}^{2}$ (range 20-25) were enrolled after written informed consent and a thorough medical examination. All subjects had normal fasting glucose and lipid values, as well as normal liver, kidney, hematological, and thyroid functions, as assessed by biochemical tests in a routine-certified laboratory (www.kimcl.at).

\section{Study design}

The study included four study sessions separated by at least 3 weeks. Participants came to the Clinical Research Center in the morning after having fasted for ca. $12 \mathrm{~h}$. They received in a randomized placebo-controlled cross-over design once placebo $(0 \cdot 9 \% \mathrm{NaCl})$, once OXT ( $1 \mathrm{pmol} / \mathrm{kg}$ per min given as an i.v. infusion in $0.9 \% \mathrm{NaCl}$ over 90 min starting at time point $-10 \mathrm{~min}$ ), once lipopolysaccharide (LPS; $2 \mathrm{ng} / \mathrm{kg}$ National Reference Escherichia coli Endotoxin, USP Convention, Rockville, MD, USA, given as an i.v. bolus at $0 \mathrm{~min}$ ), and once both OXT and LPS. Two i.v. forearm catheters were placed on the right and left forearm for infusions and blood samples respectively. Each study session lasted about $6.5 \mathrm{~h}$ and the participants remained fasted throughout the study. Blood samples were obtained at baseline (time point $-30 \mathrm{~min}$ ), at time point 0 , half-hourly during the next $2 \mathrm{~h}$, and hourly during the following $4 \mathrm{~h}$ for the measurement of ghrelin, glucose, and free fatty acids (FFA).

\section{Hormone, glucose, and FFA assays}

Plasma ghrelin concentrations were determined using a commercially available RIA kit (Peninsula Laboratories, San Carlos, CA, USA). The kit includes a rabbit polyclonal antibody against the C-terminal end of human ghrelin and $\mathrm{I}^{125}$-ghrelin as tracer, and measures total plasma ghrelin. FFA concentrations were measured using a commercially available microfluorimetric kit (Wako Chemicals, Richmond, VA, USA). Plasma glucose levels were determined using the glucose oxidase method and the Glucose analyzer II (Beckman Coulter, Inc., Fullerton, CA, USA). Data on the concentrations of plasma OXT, GH, ACTH, cortisol, and cytokines obtained from this study are published elsewhere (Clodi et al. 2008).

\section{Statistical analysis}

Data were analyzed using SPSS release 12.0.1 (SPSS, Inc., Chicago, IL, USA) as statistical software. The KolmogorovSmirnov test was used to test the normality of data. Subjects' characteristics are expressed as mean and range, circulating values of hormones and nutrients as mean \pm s.E.M. The significance of changes between different study days were assessed by repeated measurements ANOVA. The interaction between time and treatment (time $\times$ treatment) were considered the term of interest and these $P$ values are shown in the results part. When appropriate, ANOVA was followed by post hoc paired $t$-tests for comparing the intervention-induced changes at each time point. $P<0.05$ was considered statistically significant.

\section{Results}

OXT infusion lasted $90 \mathrm{~min}$ and lead to significantly elevated plasma concentrations of OXT during the first $2 \mathrm{~h}$ (Clodi et al. 2008). In accordance with previous findings (Cummings et al. 2001, Vila et al. 2007), plasma ghrelin increased during fasting (placebo sessions) and changed biphasically after LPS administration with a rapid surge at $2 \mathrm{~h}$ followed by a continuous decline afterwards $(P<0 \cdot 001$; Fig. 1A). Administration of OXT abolished the physiological increase in plasma ghrelin during fasting $(P=0 \cdot 044$, ANOVA for differences between placebo and OXT study days; Fig. 1A). Post hoc statistics identified significant changes at $60 \mathrm{~min}$ $(611 \pm 54 \mathrm{pg} / \mathrm{ml}$ in OXT sessions versus $697 \pm 52 \mathrm{pg} / \mathrm{ml}$ in placebo sessions, $P=0.013)$ and at $90 \mathrm{~min}(629 \pm 55 \mathrm{pg} / \mathrm{ml}$ in OXT sessions versus $703 \pm 57 \mathrm{pg} / \mathrm{ml}$ in placebo sessions, $P=0 \cdot 017$ ). Furthermore, OXT significantly modified the profile of changes in ghrelin following LPS administration $(P=0 \cdot 02$, ANOVA for differences between LPS and LPS + OXT study days), leading to reduced plasma ghrelin levels $2 \mathrm{~h}$ following LPS administration $(P=0 \cdot 021$, post hoc statistics; Fig. 1A). At time point $3 \mathrm{~h}$, when plasma OXT reached baseline levels, a reduced and shifted LPS-induced ghrelin peak is observed (Fig. 1A). The effects of OXT on ghrelin levels were also evaluated using the changes in ghrelin expressed as percent increase over basal values (Fig. 1B, $P<0.05$ for all above described differences). 

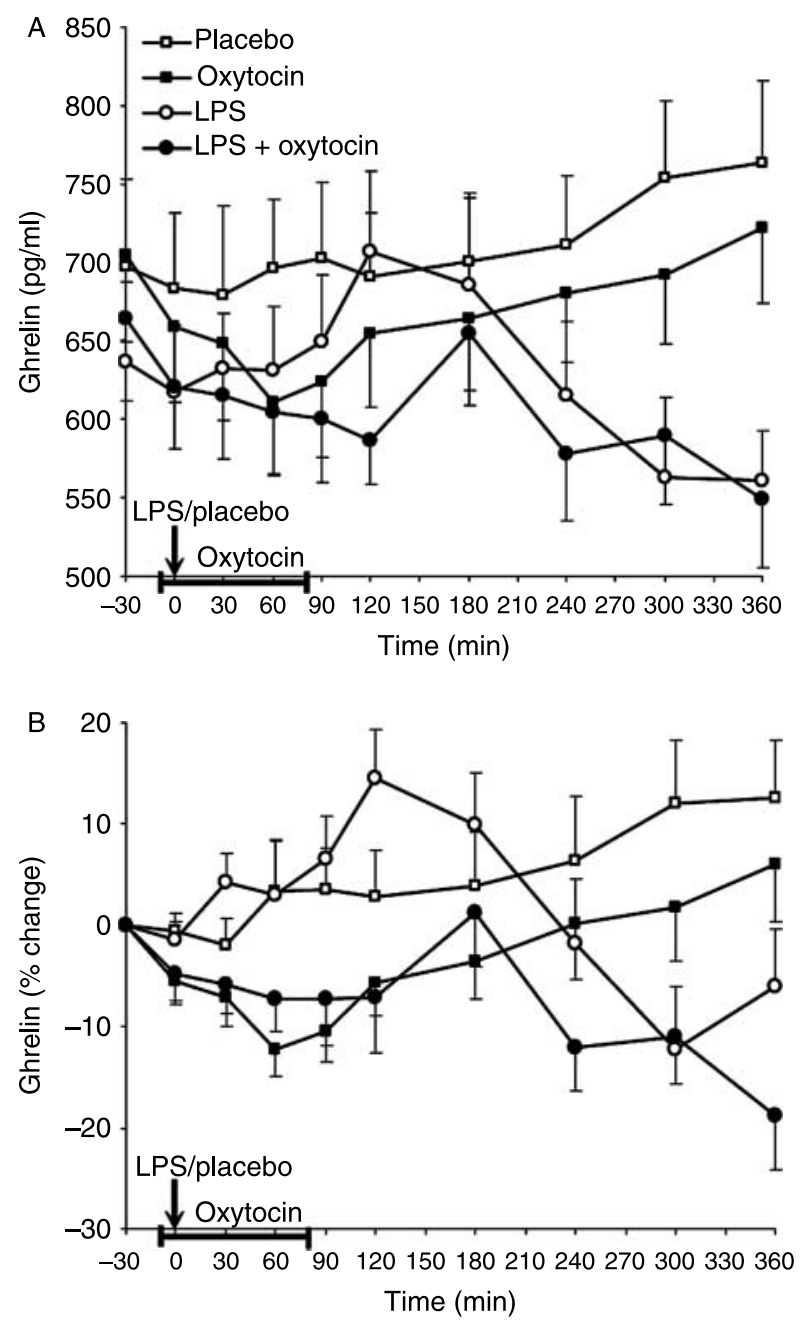

Figure 1 Plasma ghrelin concentrations in the 4 study days. (A) The changes in plasma ghrelin concentrations and (B) the changes in ghrelin as percent of basal values. LPS (2 ng/kg i.v., $t=0 \mathrm{~min}$ ) induces a rapid surge in plasma ghrelin levels at $t=120 \mathrm{~min}$ followed by a continuous decline afterwards. Oxytocin (continuous i.v. infusion of $1 \mathrm{pmol} / \mathrm{kg}$ per min between $t=-10$ and $80 \mathrm{~min}$ ) decreased both basal as well as LPS-induced ghrelin concentrations during the whole-study period. Graphs show mean \pm S.E.M. of absolute values as well mean \pm s.E.M. of changes (in percent) when compared with baseline $(t=-30 \mathrm{~min})$.

Plasma FFA levels continuously increased during fasting in the placebo days $(P<0 \cdot 001)$, and were further elevated in response to LPS $(P<0 \cdot 001$, ANOVA for differences between placebo and LPS study days; Fig. 2A). OXT increased the surge in FFA during fasting $(P=0 \cdot 005$, ANOVA for differences between placebo and OXT study days) with a significant rise at time point $5 \mathrm{~h}$ (post hoc statistics, $P=0 \cdot 017$; Fig. 2A). The administration of OXT reduced the FFA increase in response to $\operatorname{LPS}(P=0 \cdot 002$, ANOVA for differences between LPS and LPS + OXT study days) with post hoc tests showing significant changes at time points: $1 \mathrm{~h}$ $(P=0 \cdot 035), 4 \mathrm{~h}, 5 \mathrm{~h}$ (both $P<0 \cdot 001)$, and $6 \mathrm{~h}(P=0 \cdot 002$;
Fig. 2A). There were no statistically significant changes in plasma glucose concentrations between placebo and LPS days (Fig. 2B). OXT induced a small but significant elevation of plasma glucose under basal conditions $(P=0 \cdot 002$, ANOVA for differences between placebo and OXT study days) as well as after LPS administration $(P=0 \cdot 043$, ANOVA for differences between LPS and LPS + OXT study days), while post hoc tests revealed no significant changes at specific time points (Fig. 2B).

\section{Discussion}

Here, we show that systemic administration of OXT reduces plasma ghrelin concentrations under fasting conditions and also following endotoxemic stress in healthy men. During fasting, OXT increased both plasma glucose and FFA levels. During endotoxemia, OXT increased plasma glucose, but decreased the LPS-induced surge in FFA.
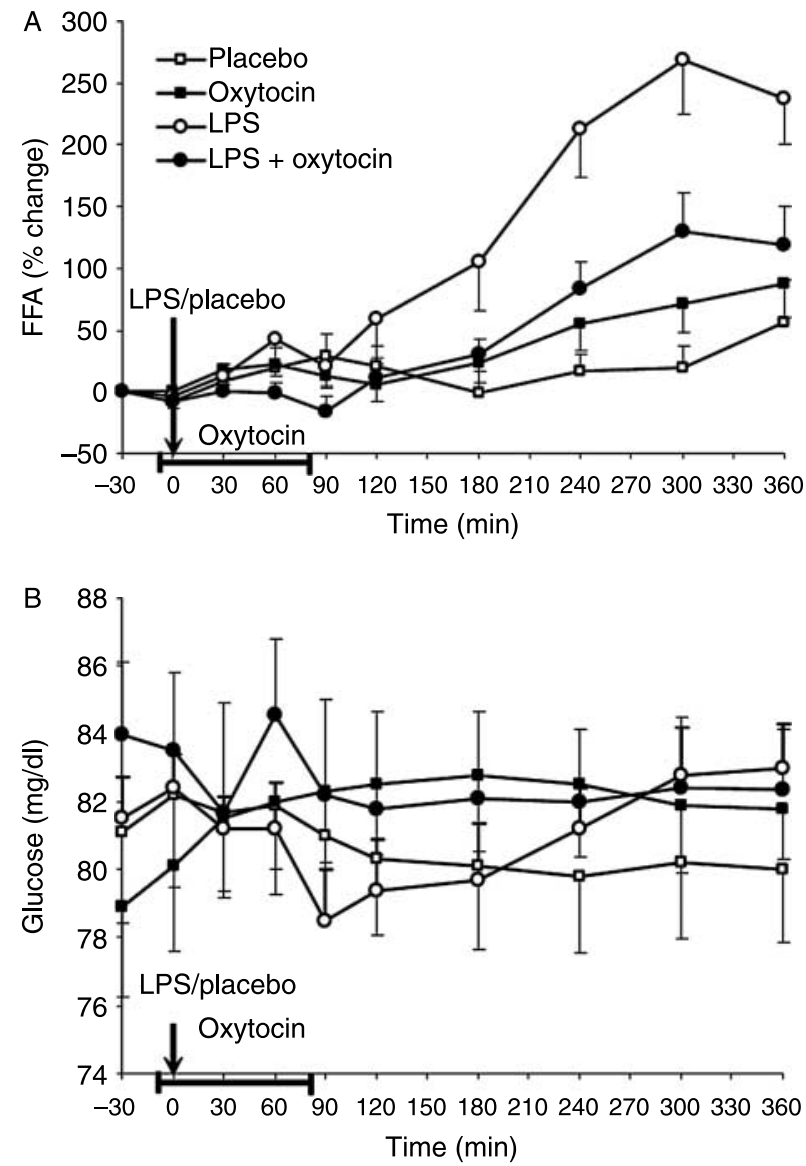

Figure 2 FFA and glucose levels in response to endotoxin and oxytocin. Plasma concentrations of (A) FFA (as percent change over the basal values) and glucose (B) in response to placebo, oxytocin (1 pmol/kg per min i.v. during $90 \mathrm{~min}, t=-10 \mathrm{~min}$ till $t=80 \mathrm{~min}$ ), LPS ( $2 \mathrm{ng} / \mathrm{kg}$ i.v., $t=0 \mathrm{~min})$, and LPS + oxytocin. Data are presented as mean \pm S.E.M. 
We recently reported that OXT had no impact on the basal levels of ACTH, cortisol, tumor necrosis factor- $\alpha$, and other cytokines, but decreased the neuroendocrine and cytokine activation during bacterial endotoxemia in men (Clodi et al. 2008). The fact that OXT not only decreased the LPSinduced ghrelin levels, but also abolished the physiological increase in ghrelin during fasting supports the existence of a strong relationship between these two hormones. The effect of OXT on the LPS-induced neuroendocrine and cytokine activation was temporary and lasted only in the presence of significantly elevated circulating OXT concentrations, which were achieved in the first $3 \mathrm{~h}$ of the study (Clodi et al. 2008). The decrease in plasma ghrelin follows a similar pattern: post hoc statistics reveals significant differences only in the presence of increased circulating OXT; and the LPS-induced surge in ghrelin seems shifted to later time points.

It is interesting to note that the i.v. administration of $1 \mathrm{pmol} / \mathrm{kg}$ per min OXT during $90 \mathrm{~min}$ increased plasma OXT levels by about threefold (Clodi et al. 2008). In human pathophysiology, OXT is increased in response to social contact, several stressors, as well as during parturition and lactation (Gimpl \& Fahrenholz 2001). Therapeutically, OXT is used to induce labor and these doses are higher than the one used in this study. One of the most important questions arising from our findings is the site of OXT actions. OXT and ghrelin have both a complex biology that should be taken into consideration when discussing the mechanisms mediating the inhibitory effect of OXT on ghrelin. Circulating ghrelin originates mainly from the stomach, and to a lesser degree from the brain, pituitary, placenta, etc (van der Lely et al. 2004). Plasma ghrelin levels are under the control of both cholinergic and adrenergic fibers (Hosoda \& Kangawa 2008). Peripherally administered OXT can bind to specific receptors distributed in several organs and throughout the gastrointestinal tract (Gimpl \& Fahrenholz 2001, Ohlsson et al. 2006). Nevertheless, there are no studies investigating the localization of the OXT receptor in ghrelin-secreting cells of the gastric mucosa. OXT can cross the blood-brain barrier and bind to OXT receptors within the CNS (de Kloet et al. 1985), as well as modify barrier properties, thereby modulating the nutrient supply to the brain (Meisenberg \& Simmons 1983). Treatment with OXT within the dorsal motor nucleus activates vagal efferent neurons increasing gastric secretion and this effect is sensitive to atropine (McCann \& Rogers 1990). I.v. administration of OXT enhances gastric and colonic motility in humans (Ohlsson et al. 2004). In summary, systemic OXT can bind to different levels within the CNS, peripheral nervous system, and within the gastrointestinal tract. The mechanisms mediating the ghrelin-inhibitory effects of OXT remain to be investigated in future studies.

Ghrelin increases the c-fos immunoreactivity of OXT neurons, which are hypothesized to mediate some of the central effects of ghrelin (Olszewski et al. 2007). OXT infusion decreases the NPY changes in response to ghrelin in men (Coiro et al. 2008). Nonetheless, the impact of OXT on ghrelin levels has not been studied. Here, we show that increases in systemic OXT lead to reductions in circulating ghrelin levels both during fasting and in response to LPS (Fig. 1). Collectively, these findings reveal an interrelated control (probably a feedback mechanism) that might be of importance in human physiology and pathology.

The cross-talk between OXT and ghrelin might be of relevance in processes where both these peptides are implicated, such as food intake, stress response, and anxiety, reproduction, cardiovascular and gastric functions (Korbonits et al. 2004, Landgraf \& Neumann 2004, Kristensson et al. 2007). Both intraventricular and peripheral administration of OXT decrease food intake (Gimpl \& Fahrenholz 2001), while both intraventricular and peripheral administration of ghrelin increase food intake (van der Lely et al. 2004). Recent research has started to unravel the mechanisms underlying the orexigenic effects of ghrelin, which seem to be dependent on hypothalamic functions (López et al. 2008). To date, there are no data on the mechanisms mediating the anorexigenic effect of OXT. Our results suggest that the decrease in circulating ghrelin might be one of the mechanisms mediating OXTinduced anorexia.

Here, we show that peripheral administration of OXT increases plasma glucose and FFA levels (Fig. 2). Systemic infusion of ghrelin induces similar effects on carbohydrate and lipid metabolism, leading to an insulin resistance state (Vestergaard et al. 2008). Therefore, OXT and ghrelin appear to have similar effects on peripheral glucose and FFA concentrations, but opposite effects on appetite regulation.

Both OXT and ghrelin increase in the presence of several acute stressors (Van de Kar \& Blair 1999, Vila et al. 2007, Lutter $e$ al. 2008) and their administration alleviates the stress response (Dixit et al. 2004, Clodi et al. 2008, Lutter et al. 2008). Ghrelin is thought to increase glucose availability to insulinindependent tissues such as the brain (Vestergaard et al. 2008). In stress situations, it is of paramount importance not to leave the provision of nutrients to chance and it is speculated that the body's ability to compensate for perturbations shifts nutrient intake towards the use of endogenous sources (Vila et al. 2007). The cross-talk between OXT and ghrelin might be involved in the control of nutrient utilization during stress and might mediate LPS-induced anorexia.

In summary, our study presents that i.v. administration of OXT reduces both basal and stress-induced systemic levels of ghrelin. The cross-talk between OXT and ghrelin might be of high importance in the regulation of energy homeostasis and stress responses.

\section{Declaration of interest}

The authors have no conflict of interest.

\section{Funding}

This study was partially funded by Research Grant 12323 of the Austrian National Bank. 


\section{Author contribution statement}

A L, M C, A van der L, and G V designed the study. G V, M R, M R, and L H performed research. All authors contributed in the analysis and interpretation of the results. G V wrote the first draft of the manuscript. All authors critically revised the manuscript and approved the final version.

\section{References}

Charmandari E, Tsigos C \& Chrousos G 2005 Endocrinology of the stress response. Annual Review of Physiology 67 259-284.

Clodi M, Vila G, Geyeregger R, Riedl M, Stulnig TM, Struck J, Luger TA \& Luger A 2008 Oxytocin alleviates the neuroendocrine and cytokine response to bacterial endotoxin in healthy men. American Journal of Physiology. Endocrinology and Metabolism 295 E686-E691.

Coiro V, Saccani-Jotti G, Rubino P, Manfredi G, Vacca P, Volta E \& Chiodera P 2008 Oxytocin inhibits the stimulatory effect of ghrelin on neuropeptide Y levels in humans. Journal of Neural Transmission 115 $1265-1267$.

Cummings DE, Purnell JQ, Frayo RS, Schmidova K, Wisse BE \& Weigle DS 2001 A preprandial rise in plasma ghrelin levels suggests a role in meal initiation in humans. Diabetes 50 1714-1719.

Dixit VD, Schaffer EM, Pyle RS, Collins GD, Sakthivel SK, Palaniappan R, Lillard JW Jr \& Taub DD 2004 Ghrelin inhibits leptin- and activationinduced proinflammatory cytokine expression by human monocytes and $\mathrm{T}$ cells. Journal of Clinical Investigation 114 57-66.

Gimpl G \& Fahrenholz F 2001 The oxytocin receptor system: structure, function, and regulation. Physiological Reviews 81 629-683.

Hosoda H \& Kangawa K 2008 The autonomic nervous system regulates gastric ghrelin secretion in rats. Regulatory Peptides 146 12-18.

de Kloet ER, Rotteveel F, Voorhuis TA \& Terlou M 1985 Topography of binding sites for neurohypophyseal hormones in the brain. European Journal of Pharmacology 110 113-119.

de Kloet ER, Joels M \& Holsboer F 2005 Stress and the brain: from adaptation to disease. Nature Reviews. Neuroscience 6 463-475.

Kojima M, Hossoda H, Date Y, Nakazato M, Matsuo H \& Kangawa K 1999 Ghrelin is a growth-hormone-releasing acylated peptide from stomach. Nature 402 656-660.

Korbonits M, Goldstone AP, Gueorguiev M \& Grossman AB 2004 Ghrelin - a hormone with multiple functions. Frontiers in Neuroendocrinology 25 27-68.

Kristensson E, Sundqvist M, Håkanson R \& Lindström E 2007 High gastrin cell activity and low ghrelin cell activity in high-anxiety Wistar Kyoto rats. Journal of Endocrinology 193 245-250.

Landgraf R \& Neumann I 2004 Vasopressin and oxytocin release within the brain: a dynamic concept of multiple and variable modes of neuropeptide communication. Frontiers in Neuroendocrinology 25 150-176.

van der Lely AJ, Tschöp M, Heiman ML \& Ghigo E 2004 Biological, physiological, pathophysiological, and pharmacological aspects of ghrelin. Endocrine Reviews 25 426-457.
López M, Lage R, Saha AK, Pérez-Tilve D, Vázquez MJ, Varela L, Sangiao-Alvarellos S, Tovar S, Raghay K, Rodríguez-Cuenca S et al. 2008 Hypothalamic fatty acid metabolism mediates the orexigenic action of ghrelin. Cell Metabolism 7 389-399.

Ludwig M \& Leng G 2006 Dendritic peptide release and peptide-dependent behaviours. Nature Reviews. Neuroscience 7 126-136.

Lutter M, Sakata I, Osborne-Lawrence S, Rovinsky SA, Anderson JG, Jung S, Birnbaum S, Yanagisawa M, Elmquist JK, Nestler EJ et al. 2008 The orexigenic hormone ghrelin defends against depressive symptoms of chronic stress. Nature Neuroscience 11 752-753.

Maier C, Riedl M, Vila G, Nowotny P, Wolzt M, Clodi M, Ludvik B \& Luger A 2008 Cholinergic regulation of ghrelin and peptide YY release may be impaired in obesity. Diabetes 57 2332-2340.

McCann MJ \& Rogers RC 1990 Oxytocin excites gastric-related neurones in rat dorsal vagal complex. Journal of Physiology 428 95-108.

Meisenberg G \& Simmons WH 1983 Minireview. Peptides and the blood-brain barrier. Life Sciences 32 2611-2623.

Murphy KG, Dhillo WS \& Bloom SR 2006 Gut peptides in the regulation of food intake and energy homeostasis. Endocrine Reviews 27 719-727.

Ohlsson B, Ringstrom G, Abrahamsson H, Simren M \& Bjornsson ES 2004 Oxytocin stimulates colonic motor activity in healthy women. Neurogastroenterology and Motility 16 233-240.

Ohlsson B, Truedsson M, Djerf P \& Sundler F 2006 Oxytocin is expressed throughout the human gastrointestinal tract. Regulatory Peptides $1357-11$.

Olszewski PK, Bomberg EM, Martell A, Grace MK \& Levine AS 2007 Intraventricular ghrelin activates oxytocin neurons: implications in feeding behaviour. Neuroreport 18 499-503.

Van de Kar LD \& Blair ML 1999 Forebrain pathways mediating stress-induced hormone secretion. Frontiers in Neuroendocrinology 20 1-48.

Vestergaard ET, Gormsen LC, Jessen N, Lund S, Hansen TK, Moller N \& Jorgensen JO 2008 Ghrelin infusion in humans induces acute insulin resistance and lipolysis independent of growth hormone signaling. Diabetes 57 3205-3210.

Vila G, Maier C, Riedl M, Nowotny P, Ludvik B, Luger A \& Clodi M 2007 Bacterial endotoxin induces biphasic changes in plasma ghrelin in healthy humans. Journal of Clinical Endocrinology and Metabolism 92 3930-3934.

Windle RJ, Forsling MR \& Guzek JW 1992 Daily rhythms in the hormone content of the neurohypophysial system and release of oxytocin and vasopressin in the male rat: effect of constant light. Journal of Endocrinology $133283-290$

Received in final form 8 June 2009

Accepted 7 July 2009

Made available online as an Accepted Preprint

8 July 2009 